Tomasz Linek - Tomasz Tanski - Wojciech Borek*

\title{
INFLUENCE OF SURFACE ROUGHNESS ON THE CAVITATION WEAR 0F P265GH AND X2CrNi18-9, STEEL CAVITATION GENERATORS
}

The aim of this paper was to determine the effect of surface roughness of cavitation generators made from two different materials: P265GH steel, with a ferritic-pearlitic structure, and X2CrNi18-9 (304L) steel with an austenitic structure on the mass loss and cavitation wear. Cavitation generators were tested in the conditions of cavitation wear environment continuously for 500 PMHs in a specially designed and constructed author's stream and flow device. Based on the carried out experiments was confirmed that the highest mass loss - $0.1752 \mathrm{~g}$ is seen for a sample of P265GH steel wet sanded with paper with the grain size of 1000. The smallest mass loss was recorded for the cavitation generator made of X2CrNi18-9 (304L) steel, sanded with sandpaper with the grain size of 2500. Certainly, the smallest number of cavitation wear effects was found for a cavitation generator made of austenitic steel X2CrNi18-9 (304L). Few places were identified based on macroscopic photographs, especially near the edges of the straight-through openings, but their number was much smaller than for the ferritic-pearlitic steel, which is associated most of all with the properties of austenitic chromium - nickel steel.

Keywords: cavitation, cavitation wear, cavitation generators, surface roughness, mass loss, P265GH steel, X2CrNi18-9 (304L) steel

\section{Introduction}

The cavitation destruction phenomenon occurs in industry wherever a quickly flowing working medium, e.g. fluid or saturated steam, is moving due to the influence of differential pressure in a technological process. The basic wear of constructional materials working in a cavitation environment consists of an abrupt change of pressure of the fluid rinsing a given surface of a constructional element of machines, devices and industrial apparats. The key methods of preventing a harmful effect of the cavitation destruction phenomenon of constructional elements are such constructional solutions as: optimum parametrisation of machine parts' geometry and the designing of a streamlined surface shape, as well as additional technological solutions such as: selection of materials and application of the adequate surface engineering treatment [1], [2], [3], [4], [5].

The purpose of this work was to examine the effect of surface roughness of the following investigated steels, i.e. P265GH and X2CrNi18-9 (304L) steel, on the mass loss and cavitation wear of cavitation generators subject to operation in a stream and flow device. Constructional elements, which were designed and modelled in advance in Computational Fluid Dynamics (CFD) numerical software, were made within the investigations [1], [2], [3]. The constructions meeting the majority of geometrical criteria were selected for further investigations in real conditions in a stream and cavitation device with the flow character of work. The selected constructional elements were made of two structural steels, the first one of $\mathrm{P} 265 \mathrm{GH}$ steel with a ferritic-pearlitic structure, and the second of $\mathrm{X} 2 \mathrm{CrNi} 18-9$ (304L) steel with an austenitic structure. The author of this work will utilise the results of the investigations and the conclusions found after the operation of steel elements for 500 operating hours [PMH - Productive Machine Hour] to implement appropriate protection of surface against cavitation wear, choosing from the available surface engineering technologies, e.g. Physical Vapour Deposition (PVD) or others [6], [7], [8], [9], [10], [11].

\section{Research material and methodology}

Following the pre-optimisation of cavitation generator dimensions and shape [1], two grades of steel were used for the purpose of investigations aimed at determination of potential places of intensive cavitation wear. The first grade is steel commonly used for pressure devices working at elevated temperatures, $\mathrm{P} 265 \mathrm{GH}$, with a ferritic-pearlitic structure, and the second is from a group of stainless steels - chromium - nickel X2CrNi18-9 (304L) steel with an austenitic structure. The P265GH steel - due to its unlimited availability and attractive, low market prices - is used for constructing heat distribution devices and heating devices, and for less important constructional parts. The X2CrNi18-9 (304L) steel, which exceeds the price of P265GH steel five times, is used for production of devices, apparats and fittings in the chemical, food, power and petrochemical industry and for constructional elements in the aviation and shipbuilding sector. The chemical composition of the structural steels, tested in the conditions of cavitation wear, is presented in Table 1.

Constructional elements made of the two steels, with their shape and dimensions selected based on the analysis of results of numerical simulations in ANSYS FLUENT software, described in

\footnotetext{
* 1,2Tomasz Linek, ${ }^{2}$ Tomasz Tanski, ${ }^{2}$ Wojciech Borek

${ }^{1}$ Odlewnie Polskie S.A, Starachowice, Poland

${ }^{2}$ Institute of Engineering Materials and Biomaterials, Silesian University of Technology, Gliwice, Poland

E-mail: tomasz.linek@odlewniepolskie.pl
} 
Table 1 Chemical composition of the structural steels tested in the conditions of cavitation wear: P265GH by PN-EN 10028:2010; X2CrNi18-9 by PN-EN 10088 [mass fraction, \%]

\begin{tabular}{|c|c|c|c|c|c|c|c|c|c|c|c|c|}
\hline \multicolumn{2}{|c|}{ Chemical composition } & $\mathrm{C}$ & $\mathrm{Mn}$ & $\mathrm{Si}$ & $\mathrm{Al}$ & $\mathrm{Cr}$ & $\mathrm{Ni}$ & $\mathrm{Cu}$ & $\mathrm{Ti}$ & $\mathrm{N}$ & $\mathrm{S}$ & $\mathrm{P}$ \\
\hline \multicolumn{2}{|c|}{ Steel } & [\%] & [\%] & [\%] & [\%] & [\%] & [\%] & [\%] & [\%] & [\%] & [\%] & [\%] \\
\hline \multirow{2}{*}{$\mathrm{P} 265 \mathrm{GH}$} & $\max$ & - & - & 0.4 & - & 0.3 & 0.3 & 0.3 & 0.03 & 0.012 & - & - \\
\hline & & 0.16 & 0.99 & 0.23 & 0.047 & 0.027 & 0.013 & 0.026 & 0.001 & 0.003 & 0.008 & 0.019 \\
\hline \multirow{2}{*}{$\begin{array}{c}\text { X2CrNi18-9 } \\
\text { (304L) }\end{array}$} & $\min$. & & & & & 17.50 & 8.00 & & & & & \\
\hline & $\max$ & $<0.03$ & $<2.0$ & $<1.0$ & - & 19.50 & 10.50 & - & - & $<0.11$ & $<0.045$ & $<0.015$ \\
\hline
\end{tabular}

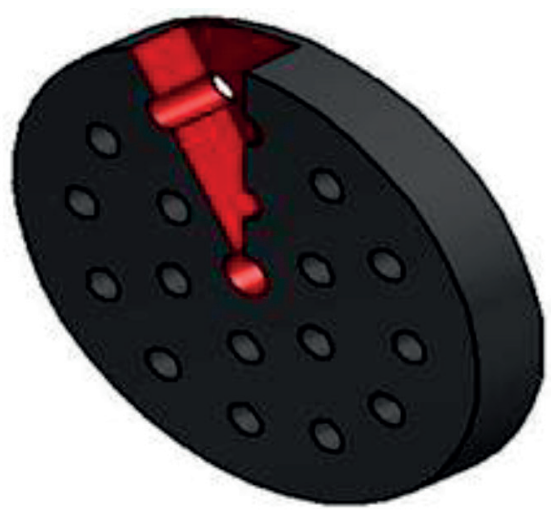

a)

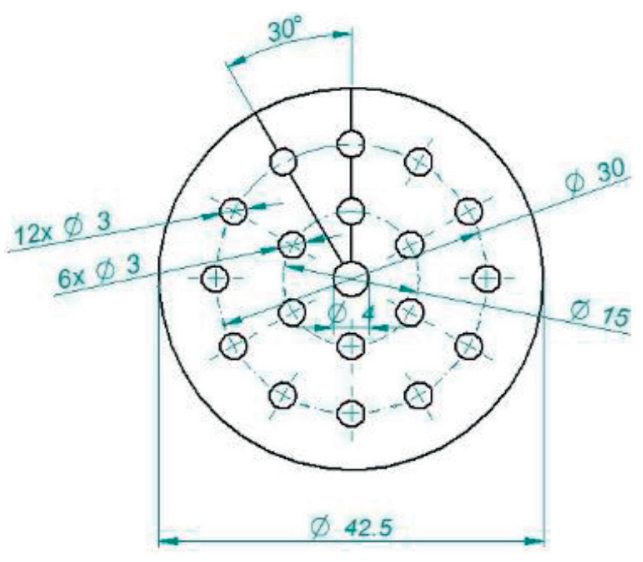

b)

Figure 1 Cavitation generator model's dimensions and shape selected based on the analysis of results of numerical simulations in ANSYS FLUENT software; cavitation generator thickness of 5 [mm], relative clearance of $P p=11.1[\%]$

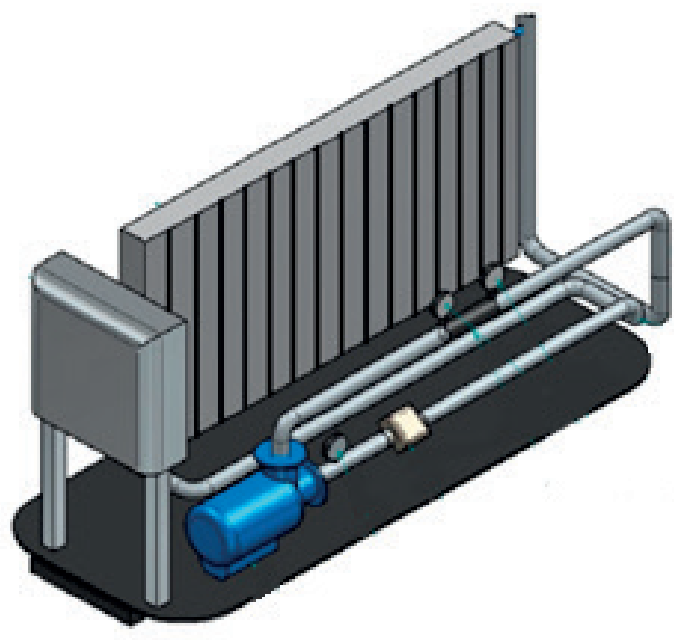

a)

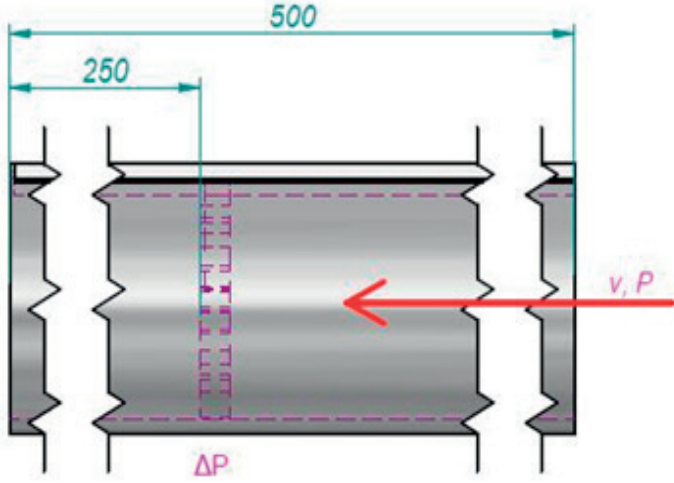

b)

Figure 2 Model of a stream and flow device generating a cavitation environment; a) isometric diagram of the device, testing and measuring system; b) simplified computer model of the cavitation generator location with the medium (water) flow direction marked

[1], shown in Figure 1, were tested in the conditions of cavitation wear continuously for 500 PMHs in a specially designed and constructed author's stream and flow device (Figure 2) generating a cavitation environment. The detailed process parameters are presented and described in [1].

The purpose of this work was to examine the effect of surface roughness of the investigated steels on the mass loss and cavitation wear of cavitation generators in a stream and flow device. Due to a large number of samples and, most of all, a long exposure time in a stream and flow device of 500 PMHs, 3 cavitation generators made of $\mathrm{P} 265 \mathrm{GH}$ steel, wet sanded with Struers sandpapers with the grain size of, respectively 200, 1000 and 2500, and one cavitation generator made of X2CrNi18-9 (304L) steel, sanded with sandpaper with the grain size of 2500 , were selected for the investigations. The samples, prior to installation in a stream and flow device generating a cavitation environment (Figure 2), as well as after 500 PMHs of continuous work in such a device, were cleaned in an ultrasound scrubber, 


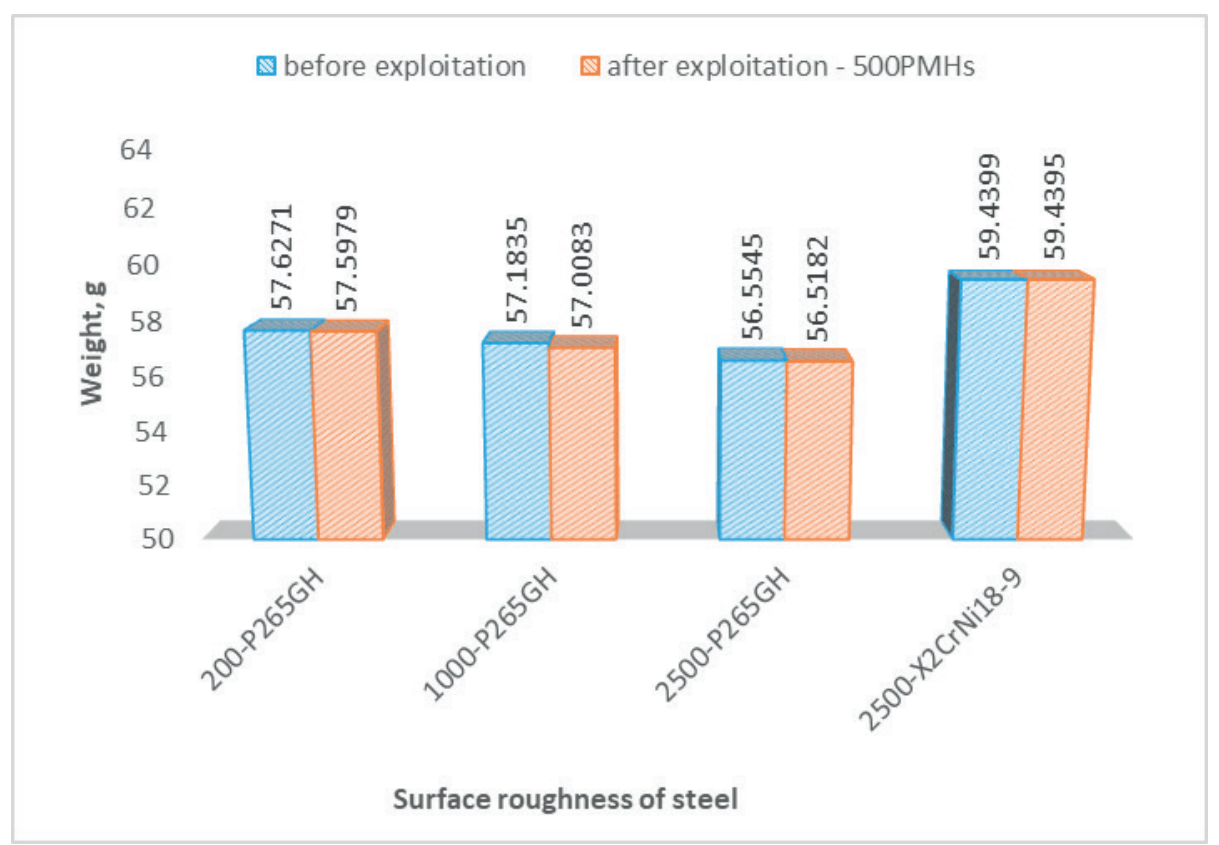

Figure 3 Loss of mass, i.e. the mass of cavitation generator made of P265GH and X2CrNi18-9 (304L) steels after use in cavitation wear conditions in a stream and flow device

and then weighed on an analytical scale, AS/X, by RADWAG. Surface roughness was determined with a Surtronic 25 contact profilometer by Taylor Hobson. At least four measurements, along the length of $16 \mathrm{~mm}$ in different areas of the cavitation generator, were made to determine the surface roughness of each sample. A motorised stereoscope microscope, Stereo Discovery V12, by Zeiss, with a magnification of 8 to $100 \mathrm{x}$, featuring an AxioVison image analysis system, was used for preliminary elimination of cavitation effect results. Detailed macroscopic examinations of the samples surface after cavitation wear were carried out with a scanning electron microscope, SUPRA 35 , at the accelerating voltage of 5 to $20 \mathrm{kV}$ using secondary electrons (SE) detection, with the magnification of $150-750 \mathrm{x}$.

\section{Results}

The cavitation generators, both before and after use in the conditions of cavitation wear in a stream and flow device continuously for $500 \mathrm{PMHs}$, were weighed and subjected to surface roughness measurements. The results of weight and surface roughness measurement examinations are shown, respectively, in Figure 3 and Figure 4.

The results of macroscopic examinations of the applied P265GH and X2CrNi18-9 (304L) steels in cavitation wear conditions made with a stereoscopic microscope, Stereo Discovery V12, by Zeiss, with the magnification of 8 to $100 \mathrm{x}$, are shown in Figure 5, Figure 6, Figure 7 and Figure 8. Meanwhile, the detailed results of examinations of the samples surface after the cavitation wear, carried out with a scanning electron microscope, SUPRA 35 , using the secondary electrons (SE) detection, are shown in Figure 9. A cavitation generator made of ferritic- pearlitic steel designated as $200-\mathrm{P} 265 \mathrm{GH}$, wet sanded with sandpaper with the grain size of 200, weighed 57.6271g before use and featured a surface roughness coefficient $R_{a}$ of 0.627 , thus falling to the 8 th surface roughness class according to PN-EN ISO 1302:2004. As a result of operating the cavitation generator in a stream and flow device for 500 PMHs, numerous corrosion centres have appeared on the generator front surface according to the flow direction of the medium, i.e. water with an inlet pressure of $244 \mathrm{kPa}$, especially along the cavitation generator sanding direction. Cavitation wear was found near the straight-through openings (Figure 5). A negligible loss of mass of approx. $0.03 \mathrm{~g}$ was also found as a result of operation and a roughness coefficient $R_{\mathrm{a}}$ fell from 0.627 to 0.41 , which is further classified as the 8 th surface roughness class. For a cavitation generator made of ferritic-pearlitic steel, designated as $1000-\mathrm{P} 265 \mathrm{GH}$, i.e. wet sanded with sandpaper with the grain size of 1000 , with the weight of $57.1835 \mathrm{~g}$ and the surface roughness class $R_{a}$ of 0.15 , which classifies it into the 10th roughness class, due to work in a cavitation environment for 500 PMHs, numerous corrosion centres have developed, especially in the area of the generator's straight-through openings (Figure 6). The highest mass loss, by as much as $0.1752 \mathrm{~g}$ in relation to all the operated generators, and the growth of the roughness coefficient $\mathrm{R}_{\mathrm{a}}$ from 0.15 to about 0.5 , was found after operating a generator marked as $1000-\mathrm{P} 265 \mathrm{GH}$, which decreases the roughness class from 10 to 8 . The last sample made of ferritic-pearlitic steel, designated as $2500-\mathrm{P} 265 \mathrm{GH}$, operated for $500 \mathrm{PMHs}$, features a surface with numerous cavitation corrosion centres on the surface (Figure 7a) and damages, breaks and flaking of the cavitation generator openings' edges were found (Figure $7 \mathrm{~b}$ and Figure 9). A small mass loss of the sample was seen for this sample from $56.5545 \mathrm{~g}$ to $56.5182 \mathrm{~g}$ and the roughness coefficient increased from 0.09 to 0.285 , i.e. declined from the 10th to 9 th roughness class.

Much better results were achieved by a cavitation generator made of austenitic steel X2CrNi18-9 (304L) wet sanded with sandpaper with the grain size of 2500 , with the weight of $59.4399 \mathrm{~g}$ and with the surface roughness coefficient $\mathrm{R}_{\mathrm{a}}$ of 0.1 , which is ascribed to the 10 th roughness class. The weight dropped 


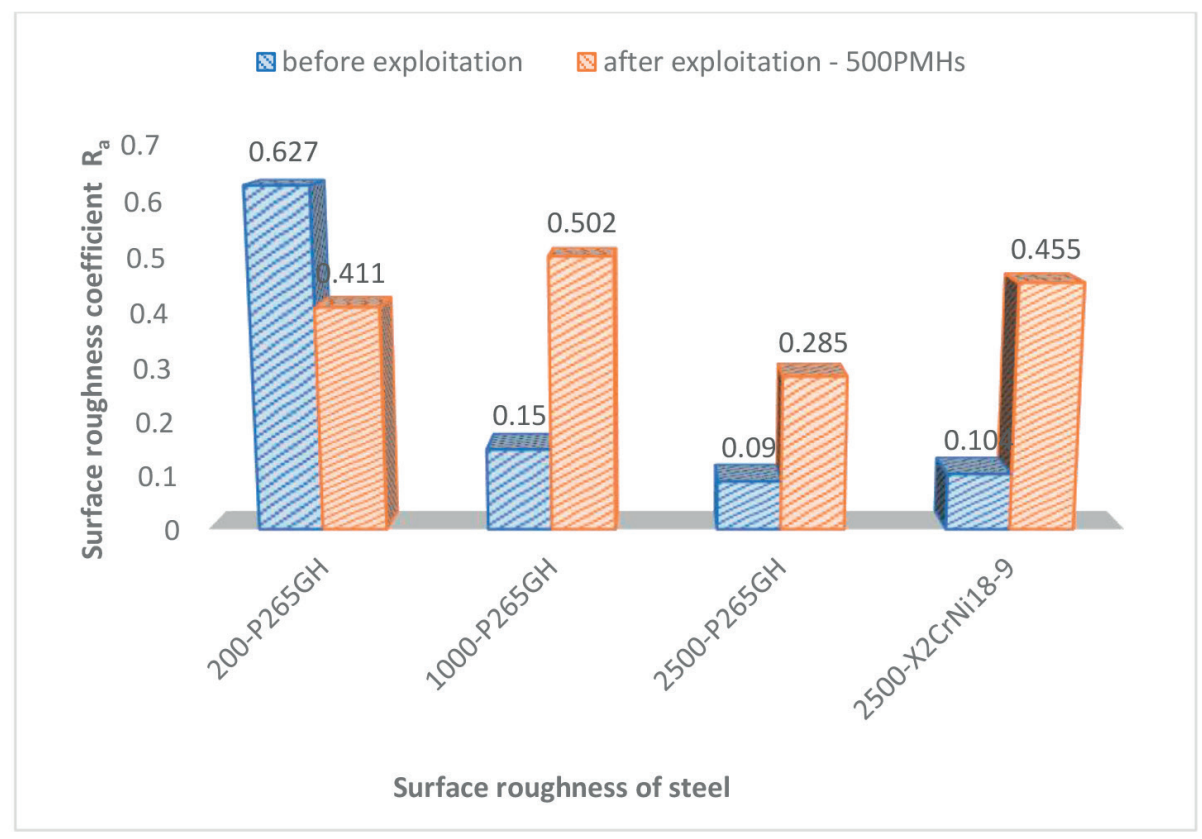

Figure 4 Variation of the surface roughness coefficient $R_{a}$ of cavitation generators made of P265GH and X2CrNi18-9 (304L) steels after use in cavitation wear conditions in a stream and flow device

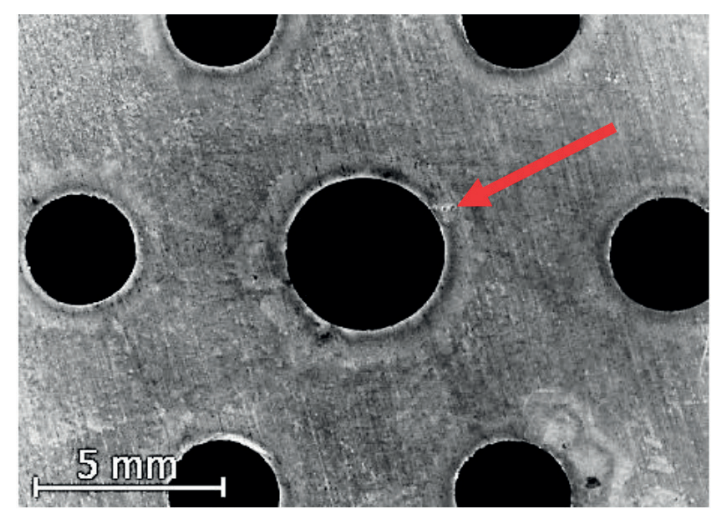

a)

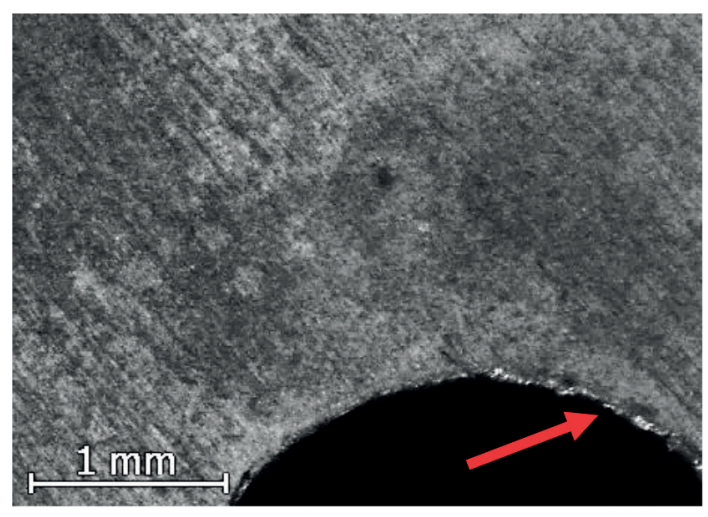

b)

Figure 5 Result of cavitation wear of the surface of a constructional element made of P265GH steel wet sanded with sandpaper with the grain size of 200 and after operation in a stream and flow device for 500 PMHs a) magnification of $8 x$; b) magnification of $40 x$

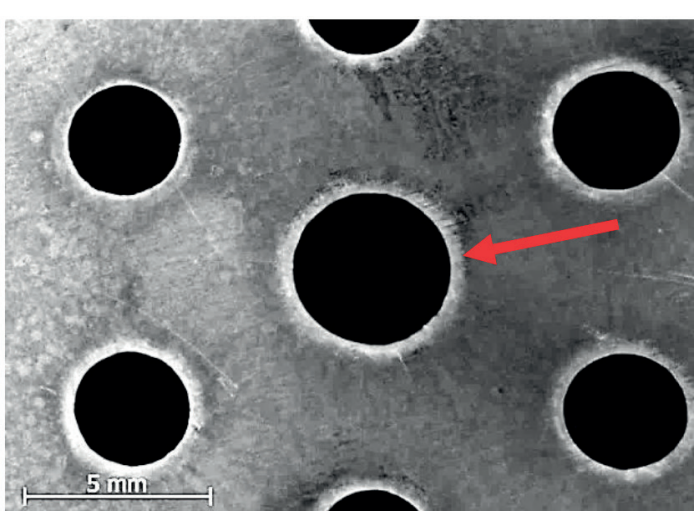

a)

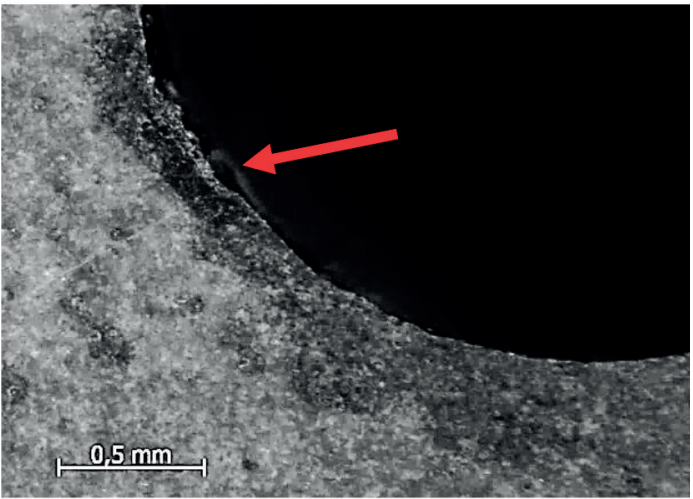

b)

Figure 6 Result of cavitation wear of the surface of a constructional element made of P265GH steel wet sanded with sandpaper with the grain size of 1000 and after operation in a stream and flow device for 500 PMHs a) magnification of $8 x$; b) magnification of $60 x$ 


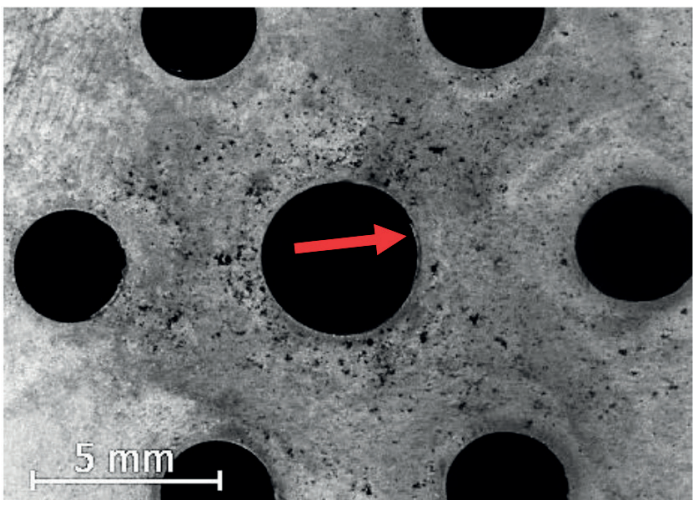

a)

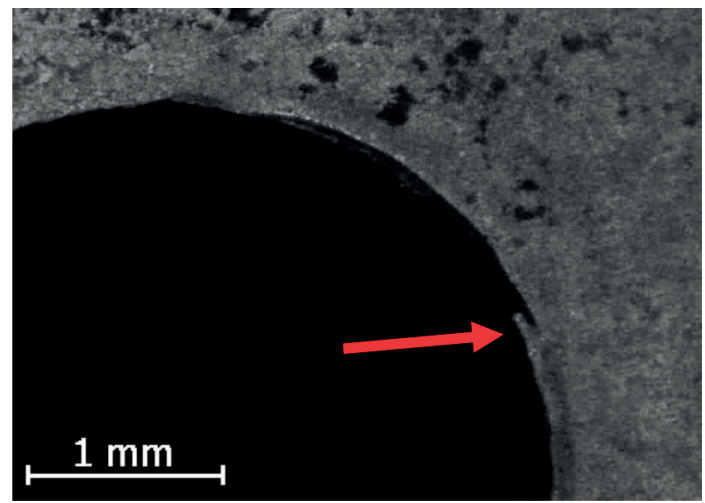

b)

Figure 7 Result of cavitation wear of the surface of a constructional element made of P265GH steel wet sanded with sandpaper with the grain size of 2500 and after operation in a stream and flow device for 500 PMHs a) magnification of $8 x$; b) magnification of $40 x$

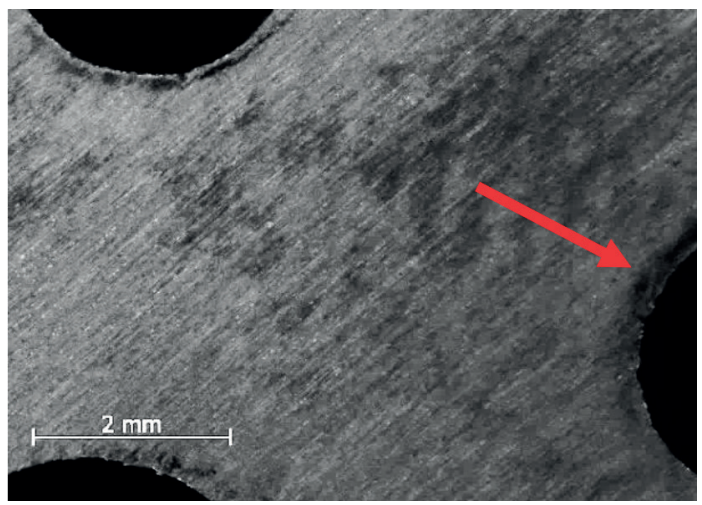

a)

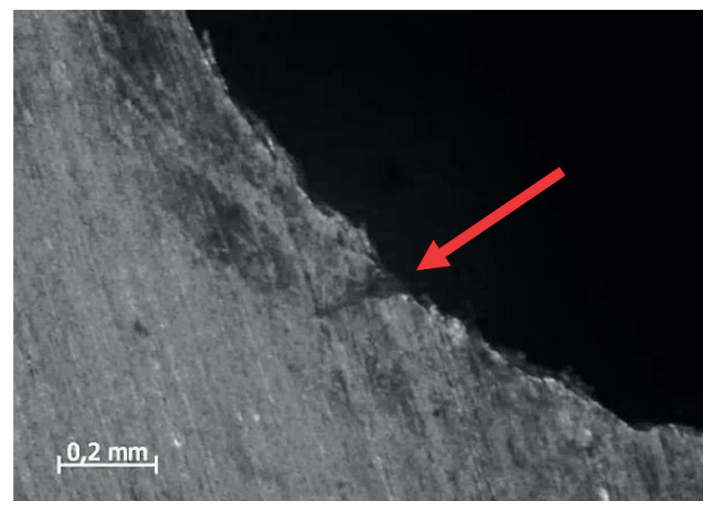

b)

Figure 8 Result of cavitation wear of the surface of a constructional element made of X2CrNil8-9 (304L) steel wet sanded with sandpaper with the grain size of 2500 and after operation in a stream and flow device for 500 PMHs a) magnification of $20 x$; b) magnification of $100 x$

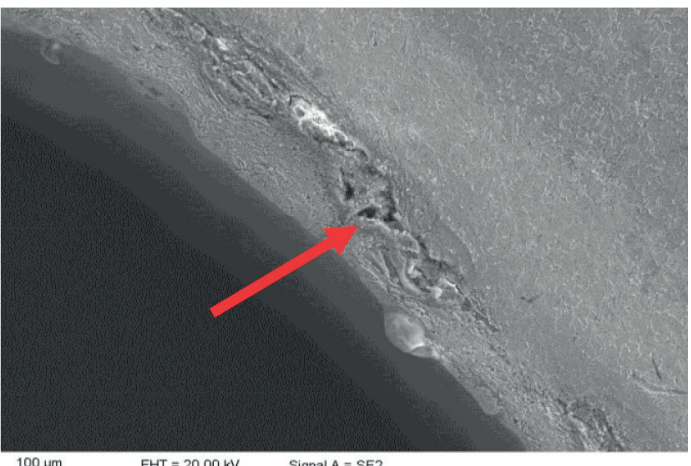

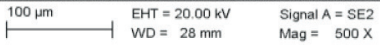

a)

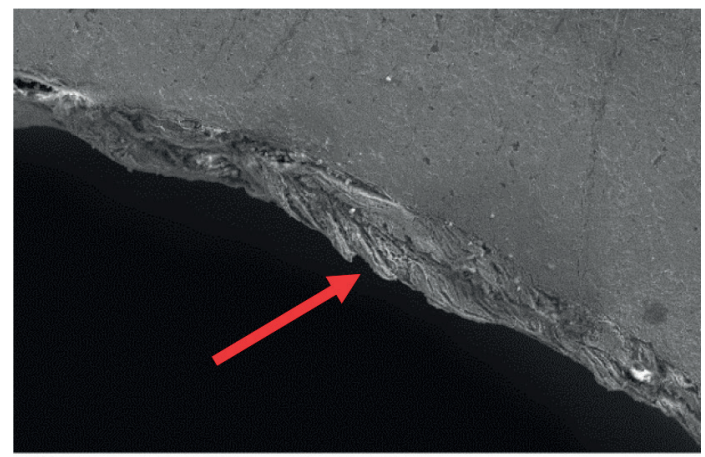

$\longmapsto \begin{array}{ll}E H T=20.00 \mathrm{kV} & \text { Signal } \mathrm{A}=\mathrm{SE} 2 \\ \mathrm{WD}=28 \mathrm{~mm} & \text { Mag }=500 \mathrm{Km}\end{array}$

b)

Figure 9 Result of cavitation wear of the surface of a constructional element made of P265GH steel wet sanded with sandpaper with the grain size of 2500 and after operation in a stream and flow device for 500 PMHs

to just $0.0002 \mathrm{~g}$ after operation, i.e. was within the measurement error range, and the roughness coefficient rose to 0.455 , which ranks it in the 8 th roughness class. Deterioration of the surface roughness with practically negligible mass loss indicates that the tested cavitation generator reached surface distortion, but no significant material losses were found, especially near the edges of the straight-through openings, but only the micro cracks. It means that the material has deformed but without losing weight, a chrome-nickel steel generator that is resistant to corrosion is characterized by better stability and resistance in conditions of the flowing medium. A few places in X2CrNi18-9 (304L) cavitation generator were identified based on macroscopic photographs, especially near the edges of the straight-through openings, where cavitation corrosion was initiated. Axial brittle cracks going deep inside the material, were also identified (pointed by an arrow, 


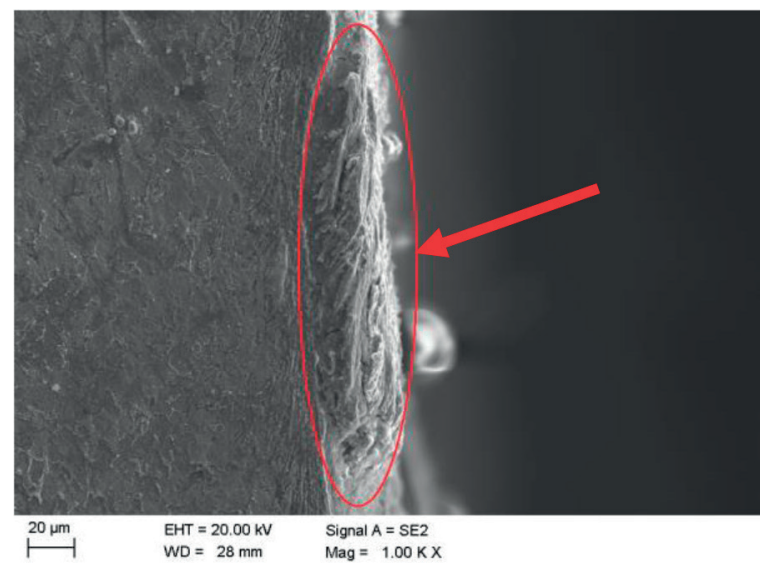

a)

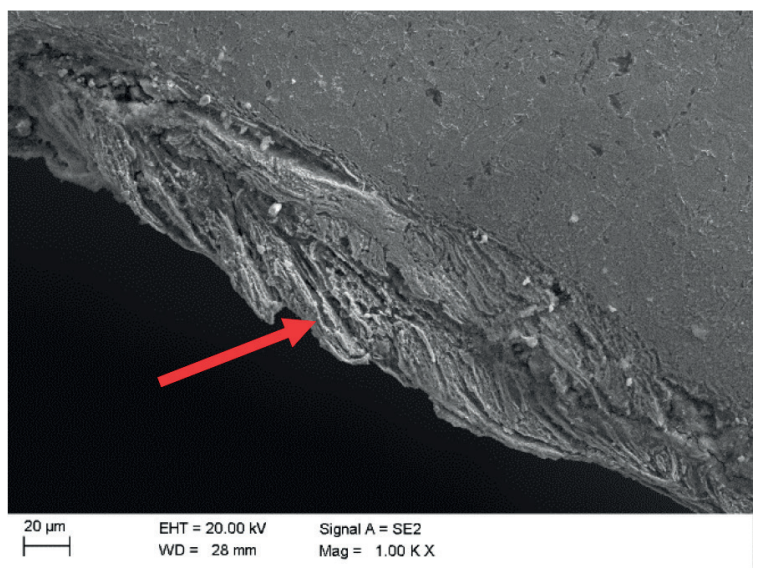

b)

Figure 10 Result of cavitation wear of the surface of a constructional element made of P265GH steel wet sanded with sandpaper with the grain size of 2500 and after operation in a stream and flow device for $500 \mathrm{PMHS}$

Figure $8 b$ ), initiated with an eroded edge of the straight-through opening.

In addition, based on the macroscopic examinations undertaken using a scanning electron microscope (SEM) for the cavitation generator made of $\mathrm{P} 265 \mathrm{GH}$ steel, it was confirmed that on the edges of the straight-through openings, especially on the edge with the biggest opening area, exist numerous places, which were being spun by the flowing water. Cavitation craters and pits were formed in the first stage, then such craters were piling up and more corrosion centres were being formed, leading to either complete damage and breaking of the material part from the edge or to material flow inside the opening according to the medium flow direction (Figure 9 and Figure 10).

\section{Summary}

Based on the experiments carried out, consisting of the operation of specially designed and produced cavitation generators in the author's stream and flow device, generating a cavitation environment continuously for $500 \mathrm{PMHs}$, made of P265GH and X2CrNi18-9 (304L) steel, it was confirmed that the highest mass loss $-0.1752 \mathrm{~g}$ is seen for a sample of $\mathrm{P} 265 \mathrm{GH}$ steel wet sanded with paper with the grain size of 1000 . The smallest mass loss, with its value at the level of the measurement error, was recorded for the cavitation generator made of X2CrNi18-9 (304L) steel, sanded with sandpaper with the grain size of 2500 . For the cavitation generators, with surface roughness in the input state of the 8th roughness class $\left(\mathrm{R}_{\mathrm{a}}=0.627\right.$ for $200-\mathrm{P} 265 \mathrm{GH}$ generator $)$, it was found that operation in a stream and flow device for 500 PMHs causes small improvements in surface quality $-\mathrm{R}_{\mathrm{a}}=0.41$.
For the generators, sanded with sandpapers with the grain size of 1000 to 2500 for the both tested steels, it was found that the surface roughness has deteriorated from the coefficient value of about $\mathrm{R}_{\mathrm{a}}=0.1$ to $\mathrm{R}_{\mathrm{a}}=0.3-0.5$, depending on the material and sanding method of generators; hence the roughness class has decreased from 10 to 9 or even 8. Numerous corrosion centres, existing on the surface of cavitation generators, especially in the P265GH steel with a ferritic-pearlitic structure, were found based on the macroscopic examinations with a stereoscopic microscope and with a scanning electron microscope. Damages, breaks and flaking of the cavitation generator straight-through opening were found in the steel.

Certainly, the smallest number of cavitation wear effects was found for a cavitation generator made of austenitic steel X2CrNi18-9 (304L). Few places were identified based on macroscopic photographs, especially near the edges of the straight-through openings, where the cavitation corrosion was initiated and also axial brittle cracks were found going deep inside the material, initiated with the eroded edge of the straightthrough opening, whereas their number was much smaller than for the ferritic-pearlitic steel, which is associated most of all with the properties of austenitic chromium - nickel steel, the basic characteristic of which is corrosion resistance. The medium pressure $(244 \mathrm{kPa})$ in a stream and flow device and the operating time have initiated the process of cavitation wear, whereas the results are not as significant as for the ferritic-pearlitic steel. The X2CrNi18-9 (304L) steel has definitely the best service life in such operating conditions, unless both engineering materials are coated with special coatings, e.g. PVD, which is planned by the authors of this publication in further research experiments, and this may largely extend the service life of cavitation generators, thus reducing the operating costs.

\section{References}

[1] LINEK, T., TANSKI, T., BOREK, W.: Numerical Analysis of the Cavitation Effect Occurring on the Surface of Steel Constructional Elements. Archives of Materials Science and Engineering, 85(1), 24-34, 2017. https://doi.org/10.5604/01.3001.0010.1555

[2] KRELLA, A., STELLER, J.: Experimental Research on the Erosion of Selected Materials Depending on the Cavitation Load (in Polish). Instytut Maszyn Przeplywowych PAN, Gdansk, 2006. 
[3] AZAR, G. T. P., YELKARASI, C., URGEN, M.: The Role of Droplets on the Cavitation Erosion Damage of TiN Coatings Produced with Cathodic Arc Physical Vapor Deposition. Surface and Coatings Technology, 322, 211-217, 2017. https://doi.org/10.1016/j.surfcoat.2017.05.050

[4] PN-EN 10028:2010 Flat Products Made of Steels for Pressure Purposes, Part 2: Non-Alloy and Alloy Steels with Specified Elevated Temperature Properties (in Polish).

[5] SZKODO, M.: Cavitation Erosion of Metal Construction Materials (in Polish). Wydawnictwo PG, Politechnika Gdanska 2008.

[6] BRENNEN, C. E.: Cavitation and Buble Dynamics. Cambridge University Press, New York, 2014.

[7] GLOWACKA, M., HUCINSKA, J.: The State of Research on Cavitation Destruction of Metal Alloys and their Protection against this Process (in Polish). Inzynieria Materialowa, 2, 79-84, 2001.

[8] SANTOS, J. F, GARZON, C. M., TSCHIPTSCHIN, A. P.: Improvement of the Cavitation Erosion Resistance of an AISI 304L Austenitic Stainless Steel by High Temperature Gas Nitriding, Materials Science and Engineering A, 382, 378-386, 2004. https:// doi.org/10.1016/j.msea.2004.05.00310.1016/j.msea.2004.05.003

[9] LI, Z., HAN, J. S., LU, J. J., ZHOU, J. S., CHEN, J. M.: Vibratory Cavitation Erosion Behavior of AISI 304 Stainless Steel in Water at Elevated Temperatures. Wear, 321, 33-37, 2014. https://doi.org/10.1016/j.wear.2014.09.012

[10] LaZArova, R., PETrov, R. H., GAYDARova, V., DAVIDKOV, A., AlEXEEV, A., MANCHEV, M., MANOLOV, V.: Microstructure and Mechanical Properties of P265GH Cast Steel after Modification with TiCN Particles. Materials and Design, 32(5), 2734-2741, 2011. https://doi.org/10.1016/j.matdes.2011.01.024

[11] PN-EN ISO 1302:2004 Geometrical Product Specifications (GPS) - Indication of Surface Texture in Technical Product Documentation (in Polish). 